\title{
Reflex memory theory of acquired involuntary motor and sensory disorders
}

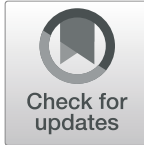

Matthias Oyigeya

\begin{abstract}
Background: Explicit and implicit memories are conserved but flexible biological tools that nature uses to regulate the daily behaviors of human beings. An aberrant form of the implicit memory is presumed to exist and may be contributory to the pathophysiology of disorders such as tardive syndromes, phantom phenomena, flashback, posttraumatic stress disorders (PTSD), and related disorders. These disorders have posed significant clinical problems for both patients and physicians for centuries. All extant pathophysiological theories of these disorders have failed to provide basis for effective treatment.
\end{abstract}

Objective: The objective of this article is to propose an alternative pathophysiological theory that will hopefully lead to new treatment approaches.

Methods: The author sourced over 60 journal articles that treated topics on memory, and involuntary motor and sensory disorders, from open access journals using Google Scholar, and reviewed them and this helped in the formulation of this theory.

Results: From the reviews, the author thinks physical or chemical insult to the nervous system can cause defective circuit remodeling, leading to generation of a variant of implicit (automatic) memory, herein called "reflex memory" and this is encoded interoceptively to contribute to these phenomena states.

Conclusion: Acquired involuntary motor and sensory disorders are caused by defective circuit remodeling involving multiple neural mechanisms. Dysregulation of excitatory neurotransmitters, calcium overload, homeostatic failure, and neurotoxicity are implicated in the process. Sustained effects of these defective mechanisms are encoded interoceptively as abnormal memory in the neurons and the conscious manifestations are these disorders. Extant theories failed to recognize this possibility.

Keywords: Unconscious memory, Reflex memory, Drug memories, Interoceptive signaling, Optogenetics, Pathophysiology, Memory erasure, Synaptic homeostasis, Defective circuit remodeling, L-type calcium channels

\section{Introduction}

Declarative (explicit) and nondeclarative (implicit) memories are forms of long-term memories that guide the daily activities of human beings. It is suspected that there exists a pathological form of implicit memory, thought to be an "aberrant memory" responsible for excitatory involuntary and uncontrollable motor and sensory phenomena disorders such as Tardive syndromes,

Correspondence: droyigeya@gmail.com

Department of Psychiatry, Federal Medical Centre, Makurdi, Benue State, Nigeria phantom phenomena, flashback, posttraumatic stress disorders (PTSD), and related disorders. Because of the assumption that these disorders are all acquired, have similar characteristics, and originate from defective circuit remodeling and their persistence are connected to a pathological form of memory, they are herein categorized as "acquired reflex phenomena disorders" (ARPDs). These disorders have been problems for both patients and physicians for centuries.

All extant pathophysiological theories have failed to provide basis for effective treatment of tardive
SpringerOpen

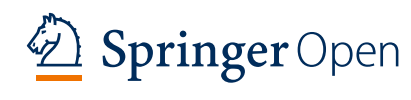

(c) The Author(s). 2021 Open Access This article is licensed under a Creative Commons Attribution 4.0 International License, which permits use, sharing, adaptation, distribution and reproduction in any medium or format, as long as you give appropriate credit to the original author(s) and the source, provide a link to the Creative Commons licence, and indicate if changes were made. The images or other third party material in this article are included in the article's Creative Commons licence, unless indicated otherwise in a credit line to the material. If material is not included in the article's Creative Commons licence and your intended use is not permitted by statutory regulation or exceeds the permitted use, you will need to obtain permission directly from the copyright holder. To view a copy of this licence, visit http://creativecommons.org/licenses/by/4.0/. 
syndromes [1-3], flashbacks and PTSD [4-7], and phantom phenomena [8-13].

The objective of this article is to propose an alternative pathophysiological theory that has potentials for new interventions. The author sourced over 60 journal articles that treated topics on memory, and involuntary motor, and sensory disorders, from open access journals using Google Scholar, and reviewed them, and this helped in the formulation of this theory. From the reviews, the author believes that physical or chemical insult to the nervous system could induce defective circuit remodeling. This may lead to generation of a variant of implicit (automatic) memory, herein called "reflex memory," which is encoded interoceptively to cause these phenomena states.

Reflex memory theory presumes that when there is an insult to nervous system due to physical injury or chemical assault, the neural cells in an effort to remodel by reestablishing connectivity or restoring lost cells trigger complex hyperexcitatory molecular and structural neural mechanisms that overwhelmed the neural homeostatic capacity and this may lead to excitotoxicity. When this state is sustained for a long time, the affected cell ensembles in the network encode this state interoceptively as a memory, an aberrant memory that is labile, unstable, and in a "persistent recall mode," manifesting as excitatory involuntary and uncontrollable motor and sensory phenomena disorders (the interoceptive awareness).

The author proposes that the formation of this aberrant memory, like the normal memory, is assumed to involve phases of acquisition, consolidation, and retrieval but, unlike normal memories, it fails to reconsolidate, rather it remains in "persistent retrieval mode." The author believes that understanding this type of memory may equally lead to the understanding of the origin and pathophysiology of these distressful phenomena states, which in turn will guide researchers toward formulation of effective therapeutic options. The author thinks too that the nature of this type of memory may not be different from what have been variously referred to as maladaptive memory or dysfunctional memories [14], drug memories [15-17], proprioceptive memories [13], involuntary memories [4], pathological memories [18], and intrusive memories $[18,19]$. Because this type of memory is in a perpetual recall mode, it is vulnerable to interferences from pharmacological, molecular, optogenetic, and behavioral manipulations.

In the main text, the author highlighted on circuit remodeling to create an understanding of how a defect in remodeling can cause an end-state disorder. An overview of memory was given but with focus on acquired implicit (automatic) memory to lay a foundation for the explanation of the concept of Reflex Memory. The discussion continues with the explanation of the reflex memory theory, its proposed role in the pathophysiology of "acquired reflex phenomena disorders," and its applications in proposing treatment options for the disorders.

\section{Main text}

This section and its subsections explain how "reflex memory" contributes to the persistence of excitatory acquired involuntary motor and sensory disorders despite all forms of treatment. Acquired involuntary motor and sensory disorders such as Tardive syndromes, phantom phenomena, flashback, posttraumatic stress disorders (PTSD), and related disorders are intractable, even with treatment. In this main text, the author hypothesized that the intractable nature of these disorders is due to encoding of maladaptive (reflex) memory secondary to maladaptive synaptic plasticity that originates from defective circuit remodeling. The role of defective circuit remodeling in acquired involuntary motor and sensory disorders is discussed. An overview of the role of memory in acquired involuntary motor and sensory disorders is also discussed. The process of formation of maladaptive (reflex) memory from implicit memory is described. A highlight of the applications of reflex memory theory in the treatment of the disorders is also given.

\section{Role of defective circuit remodeling in acquired involuntary motor and sensory disorders Normal circuit remodeling}

Circuit remodeling is a normal neurodevelopmental process of neuronal regeneration or regrowth. The formation of adult neuronal circuitry often involves generating too many connections of axon, dendrite, and synapse, most of which are not necessary and are later eliminated with high temporal and spatial resolution. This is called circuit remodeling and it is a conserved strategy to refine and sculpt neural circuits during the assembly of vertebrate and invertebrate nervous systems [20]. The hallmark of developmental circuit formation is that postsynaptic cells are initially contacted by many presynaptic partners before synaptic elimination processes remove excessive connections and establish mature connectivity [21].

\section{Defective (pathological) circuit remodeling}

In adult humans, remodeling occurs following physical or chemical insult to the nervous system in an attempt to reestablish connections, but sometimes appropriate connectivity fails, and when this happens, it results in a disease state. A typical example is the reported dyskinesia induced by chronic L-DOPA treatment as a result of aberrant rewiring of cortico-striatal synapses on medium spiny neurons (MSNs) of the striatum [22]. 
Fine sensory perception and intricate motor control are orchestrated by a discrete and permanent communication between the central nervous system (CNS) and the parasympathetic nervous system (PNS), and this cross-talk between the CNS and PNS is critical for integration of sensory inputs, being mechanical, auditory, gustatory, and olfactory, which are all relayed to the CNS through the nerves [23]. During neural perturbation, this communication can be disturbed, and if appropriate connectivity fails, the resultant effect is maladaptive response which in this context can be a disturbance of Melzack's "neuromatrix" and "neurosignature" (culled from: $[9,10,13])$. The neurophenotypes of this maladaptive response is the observed involuntary and uncontrollable sensory disorders such as phantom syndrome, flashback, and PTSDs.

The neuromatrix can be conceptualized as a network of neurons within the brain that integrates numerous inputs from various areas including somatosensory, limbic, visual, and thalamocortical components [10]. The term "neurosignature" refers to the output patterns of the neuromatrix, which are specific for an individual and are continuously being updated based upon one's conscious awareness and perception of the body and self as well as epigenetic changes $[9,10,13]$.

Studies have revealed that tissue or nerve injury triggers or induces maladaptive plastic changes in peripheral nociceptive nerve endings, in the dorsal horn of the spinal cord, as well as in supraspinal and cortical areas $[9,10,13,24-26]$, causing excessive aberrant inputs [13], and these have been implicated in chronic pain syndromes and phantom limb pains. The remodeling of supraspinal axonal circuits mediates functional recovery after spinal cord injury, and this process critically depends on the selection of appropriate synaptic connections between cortical projection and spinal relay neurons [21].

The plastic changes affect the morphology and number of neurons, followed by increased neuronal activity, expansion of the neuronal receptive field, and hyperexcitability $[9,10,13]$. The sum effect could be augmentation of the memory storage capacity of neurons, as structural rewiring allows more variability and therefore a larger number of potential circuits to be generated, implying a larger memory capacity per synapse [24]. In the process, the system may be overwhelmed by the high neural current generated and lose its homeostatic control.

While pruning, remodeling and synaptic connections between neurons occur, those neurons within affected networks must make homeostatic responses to reestablish a proper balance of excitation and inhibition, because normal cognition and behavior depend on tight neuronal homeostatic control mechanisms. Loss or gain of function of any protein that alters synaptic output may cause neurological or psychiatric phenotypes because changes in neuronal excitability demand compensation, which may, in turn, exhaust the homeostatic capacity of the network [27]. Failure to maintain tight neuronal homeostatic control may lead to pathological synaptic remodeling.

The L-type calcium channels (LTCCs) are known to play an important role in triggering intracellular cascades related to synaptic plasticity and in Hebbian synaptic plasticity at glutamatergic synapses [28], and are involved in normal brain development and plasticity [29]. However, dysregulated Cav 1.2 and Cav1.3 isoforms of LTCCs (the only LTCCs present in the brain) leading to calcium overload have been variously cited to be implicated in pathological circuit remodeling and manifesting as neuropsychiatric disorders such as bipolar disorder (BD), schizophrenia (SCZ), major depressive disorder, autism spectrum disorder, attention deficit hyperactivity disorder, and drug dependence-associated symptoms [29-35]. Defects in remodeling have been attributed to a variety of psychoneurological conditions [20]. Synaptic disease hypothesis holds that a diseasedefining experience would trigger specific forms of synaptic plasticity, which in susceptible subjects would become persistent and lead to the disease [36]. Current models of addiction and anxiety, Autism, schizophrenia, synesthesia, and a host of other neuropsychatric disorders stem from the idea that aberrant function and remodeling of neural circuits cause the pathological behaviors $[20,36]$. The author believes that all the disorders that fall within the spectrum of acquired reflex phenomena disorders are synaptic disorders. The author thinks the aberrant growths coupled with inappropriate connectivity are capable of generating hyper-excitable ectopic neural current, resulting in spontaneous discharges, high enough to escape neural homeostasis. If this abnormal neural activity is sustained for a long time, it could be saved as an aberrant memory (reflex memory) and may continue to be recalled automatically, manifesting as end point disorders such as Tardive syndromes, phantom phenomena, flashback, posttraumatic stress disorders (PTSD), and related disorders. Merlo and colleagues [14] noted that the persistent nature of maladaptive memory or dysfunctional memories is a common characteristic in several psychiatric disorders including posttraumatic stress disorder (PTSD), specific phobias, and drug addiction.

Lüthi and Lüscher [36] lamented that even though the notion of synaptic diseases has received much attention, no candidate disorder has been sufficiently investigated to yield new, rational therapies that could be tested in the clinic. Reflex memory theory, as circumstantial and speculative as it may seem, promises to offer an 
alternative explanation to the pathophysiology and treatment of ARPDs.

\section{Role of memory in acquired involuntary motor and sensory disorders Definitions of memory}

Memory can be defined as processes involved in the acquisition, storage, retention, and later retrieval of information. In simplistic terms, acquisition can be defined as the encoding of new information, consolidation is the stabilization of the new information for storage, retention is the preservation of the information, and retrieval is the later recall of the information [37]. It is also the ability to retain information and recall it at a later time, which is a biologically fundamental function essential for survival [18]. It is considered to be a process that has several stages, including acquisition, consolidation, retention, retrieval, and performance [38]. Retrieval is the only possible measure of memory performance and it is of paramount importance in determining our uniqueness as individuals [39]. Furthermore, memories shape our identity: we are who we are because of our memories, which guide our thoughts and decisions, and also influence our emotional reactions [18]. They are crucial to the cognitive essence of who we are as human beings [40]. Broadly, memory phenomena have been categorized as explicit or implicit; explicit memories for experience involve the hippocampus-medial temporal lobe system, and implicit basic associative learning and memory involves the cerebellum, amygdala, and other systems [41].

\section{Classifications of memory}

Short term versus long term Memory is broadly classified into short term and long term. Short-term memory has capacity of between 5 and 9 items and can hold information for approximately 20 to $30 \mathrm{~s}$ [18, 37, 42]. Short-term memory is formed and stored in the hippocampus and later consolidated in extra-hippocampal structures as long-term memory. Figure 1 shows types of memory and how memory transforms from simple stimulus to long term memory. Long-term memory has an almost infinite capacity, and information in longterm memory may stay there for the duration of a person's life [18, 37, 42]. Long-term memories are stored throughout the brain as subsets of neurons that probably fire together in the same pattern each time [40].

Implicit (nondeclarative) versus explicit (declarative) Long-term memory can be sub-classified into implicit (nondeclarative) and explicit (declarative) memories based on retrieval process as shown in Fig. 2. Explicit (declarative) memory or episodic memory is also called conscious memory. It is conscious, intentional remembering of information and is the most familiar system of recall of factual information such as dates, words, faces, events, and concepts $[18,43]$. Declarative memory requires a concerted effort to bring to the surface. Implicit (nondeclarative) memory is sometimes referred to as unconscious memory or automatic memory; it requires little if any effort to recall, and it is a collection of abilities that are expressed through performance (motor skills and perceptual skills) without requiring conscious memory content $[42,43]$. It uses past experiences to remember things without thinking about them. Procedural memory, which is a subset of implicit memory, is a part of the long-term memory responsible for knowing how to do things, also known as motor skills. It is recall of how to do things such as swimming or driving a car [18, 43]. They can be motor memories of skills and habits in which skeletal muscles are involved or sensory memories involving senses of smell, sight, taste, or touch. Others are senses such as proprioception, which helps us in climbing/walking down staircase without looking at each step [44], or equilibrioception which helps in knowing that there is a change of direction in a moving vehicle even with eyes closed, and a host of others that are also consolidated unconsciously in long-term memories. However some other structures are also suspected to be involved in the formation of unconscious memory. Functional magnetic resonance imaging of PTSD patients revealed increased activation in sensory and motor areas including the insula, precentral gyrus, supplementary motor area, and mid-occipital cortex [5]. Furthermore, amygdala is also involved in some forms of acquired implicit memories called METH-associated memory or visceral memories [17], or flashbulb memory [45], and the parietal lobe is suspected to be involved in the formation of some form of acquired implicit memories, the proprioceptive memories [13]. The implicit

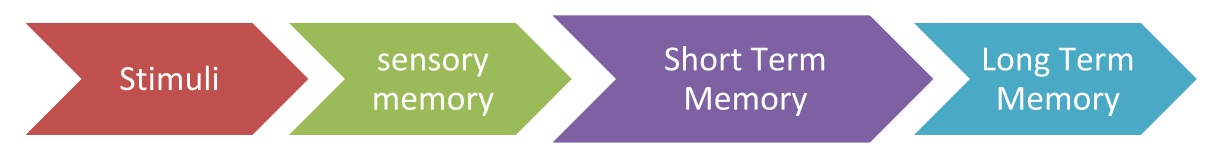

Fig. 1 Simple process diagram showing types of memory, and their transformations. Whatever information that is picked from the environment by the senses is quickly transferred into short-term memory in the hippocampus. Short-term memory is not consolidated; rather it is processed to long-term memory in the hippocampus which in turn is consolidated in extra-hippocampal structures. The different colors in the figure simply represent different types of memory and how memory transforms from simple stimulus to long term memory 


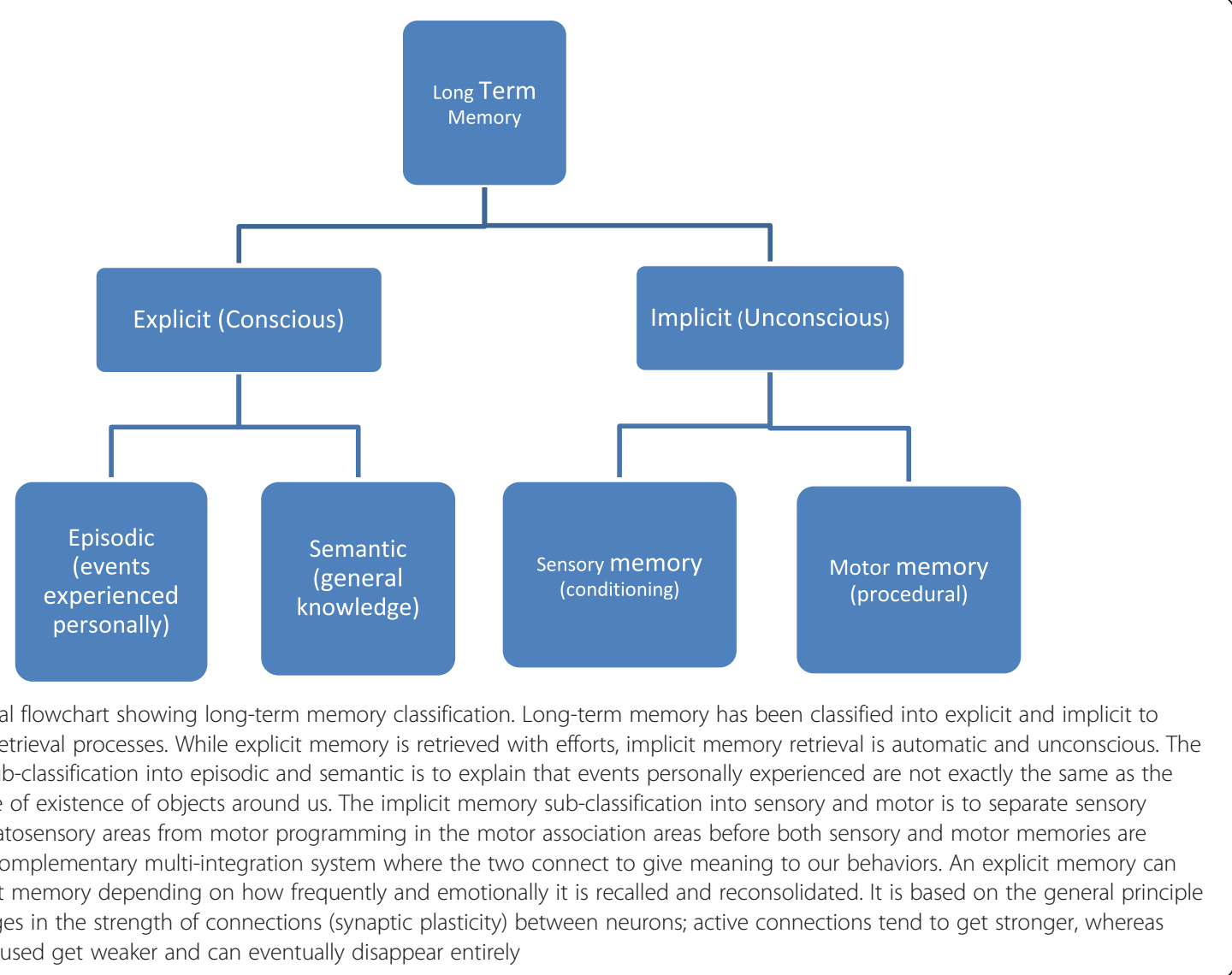

memory can be sub-classified into sensory and motor to separate sensory processing in somatosensory areas from motor programming in the motor association areas before their integration in the complementary multiintegration system where the two connect to give meaning to our behaviors.

Acquired versus genetic Implicit memory can also be sub-classified into acquired and genetic. Acquired implicit memories are our learning and personal experiences as we interact with our environments (internal and external) as well as those we inherit epigenetically from our parents through developmental process that has formed the basis for the concept of Developmental Origins of Health and Disease (DOHaD) [46-52]. Some acquired implicit memories are somatic and limited to the individual, and not passed on to the offspring. Adaptive immune response (adaptive immunity) is thought to be an example of acquired implicit somatic memory, so also delayed reaction to neuroleptic drugs.

Genetic memory is a memory present at birth that exists in the absence of motor or sensory experience, residing in specific molecules of deoxyribonucleic acid. Information is retrieved by transcribing the DNA message in the form of ribonucleic acid and then translating the RNA message into protein [53]. It is stably inherited through a process of cell division (meiosis) and transferred from generation to generation.

\section{Normal memory formation processes}

Normal memory formation involves adaptive synaptic plasticity, relying on learned associations, memories, and emotions and partly associative in a Hebbian fashion (rapidly induced and input specific).

Encoding Generally, forming a memory involves encoding, storing, retaining, and subsequently recalling information and past experiences [42]. The five traditional senses recognized in humans and considered in explaining encoding are all exteroceptive (the perception of environmental stimuli acting on the body). The ability to detect other stimuli beyond those governed by these most broadly recognized senses also exists, and these sensory modalities are interoceptive (any of the senses that detect conditions within the body). Takeuchi and colleagues [54] noted that during memory formation synaptic plasticity is induced at appropriate synapses and that this is dependent on the type of activity happening at the time. By implication, this can be external activity sensed by sight, smell, hearing, touch, or taste or internal 
activities through interoceptive signaling. Encoding through interoceptive pathway is certainly different from that of the exteroceptive pathway. Hirst and Phelps [45] in an attempt to explain the seemingly distinctive character of flashbulb memories (FBMs) also observed that a set of encapsulated mental processes govern the encoding, retention, and retrieval of FBMs, different from other autobiographical memories, or any other type of memory. It is therefore logical to reason that encoding cannot be limited to the five traditional senses.

The focus of this article in an attempt to explain reflex memory is on interoceptive sense which is viewed as a multimodal integration not restricted to any sensory channel, not restricted to mere sensations, but also relying on learned associations, memories, and emotions and integrating these in the total experience which is the subjective representation of the body state [55]. Price and Hooven [56] and Cameron [57] have explained interoception as perception of sensations from inside the body and to include the perception of physical sensations related to internal organ function such as heart beat, respiration, satiety, and the autonomic nervous system activity related to emotions. They opined that much of these perceptions remain unconscious; what becomes conscious, that is, interoceptive awareness, involves the processing of inner sensations so that they become available to conscious awareness.

Even though the perspective of these authors in the usage of the word "interoception" in their review is not exactly the same with the sense the author is using it here, some features such as the perception involves "nervous system," "sensations from inside the body," and "perceptions remain unconscious" are shared in the same sense. The author therefore thinks that there are more types of encoding than presently thought since encoding is about perception through a sensor, learning what was perceived, and relating it to past knowledge.

Flowing from the above synthesis, some unconscious encodings, more refined and deeper than the known traditional encodings, may exist through the process of interoception and may have accounted for the proposed reflex memory. The process is assumed to begin with the creation of a memory trace or engram in response to biochemical stimuli, causing complex excitatory synaptic responses that are encoded as a memory which is unconscious, labile, and reflexive and also partly associative in a Hebbian fashion (rapidly induced, and input specific). Hebbian forms of plasticity, such as LTP, rapidly modify the efficacy of individual synapses associatively in an input-specific manner, and they are thought to represent the cellular mechanisms for storing memories [58].

The average human brain houses about 86 billion neurons [59] with each connecting with neighboring neurons to form elaborate networks that generate neural-wide actions. Hoshiba and colleagues [40] noted that accumulating evidence has suggested that memories are stored as a subset of neurons that probably fire together in the same ensemble and that memories are encoded and recalled in the form of synchronous activation of cell ensembles. They further noted that cell ensembles are organized by synaptic ensembles, which can allow the brain to form an astronomical number of connections and that the defined circuits responsible for each behavior connect with one another in order to produce executional hierarchies. For this reason, an insult at a location may create an effect in another point distant from site of insult, and so, a lot of CNS disorders are network disorders.

Molecular and structural mechanisms Long-term memory formation is accompanied by structural and functional changes of neural networks that require de novo gene expression [18]. According to Tong and colleagues [60], memory mechanisms are heterogeneous in form, structure, and time course, yet exhibit many commonalities across regions of the brain. These mechanisms can be separated into two broad categories: molecular, which includes intracellular cascades, molecular signaling, neuromodulatory influences, activitydependent protein synthesis, and epigenetic modifications, and structural, which includes physiological changes such as long-term potentiation or other synaptic weight modifications, alterations to neuronal morphology such as dendritic branching, changes to terminal shapes or numbers, and ancillary modifications such as effects on glia or cell adhesion to the extracellular matrix, as well as changes to neuron number via adult neurogenesis or selective apoptosis [60]. From genetic, molecular, electrophysiological, and anatomical investigations, it has emerged that molecular modifications at all levels (post-translational, translational, and transcriptional) play a critical role in long-term memory formation and these changes include both general mechanisms of long-term plasticity, which occur in many brain regions involving long-lasting changes, and selective mechanisms, which are found in specific brain regions and cell populations [18], or cell ensembles [40] or microdomains [54].

LTM memory formation is dependent on protein kinase G (PKG), protein kinase C (PKC), calcium/calmodulin-dependent protein kinase II (CaMKII), ionotropic glutamate NMDA receptors complexes, and phosphorylation of cyclic AMP (cAMP) response element binding protein (CREB), and has long been associated with persistent structural changes in specific brain regions involved in the formation of memory [60]. It has emerged that after learning glucocorticoid receptors (GRs) 
regulate several intracellular signaling pathways known to be required for memory consolidation of stress related events $[18,61,62]$. These include the pathways activated by CREB, mitogen-activated protein kinase (MAPK), calcium/calmodulin-dependent protein kinase II (CaMKII), and brain-derived neurotrophic factor (BDNF), so BDNF-dependent pathway is a key downstream effector of GR activation during memory consolidation [18]. Brain-derived neurotrophic factor is a potent mediator both of synaptic plasticity on the cellular level, and of the interaction of an organism with its environment on the behavioral level, and has been confirmed to be a protein-synthesis-dependent memory enhancer [63]. Therefore, BDNF-dependent pathway is a strategic therapeutic target of involuntary memories connected to emotion such as PTSDs. Endocannabinoid system also plays an important role in modulating consolidation and reconsolidation of emotionally aversive memories through a combined action of the type 1 cannabinoid receptor (CB1R) and glucocorticoids [18].

One general feature of long-term memory formation across memory systems and species is that a newly encoded memory initially exists in a fragile state, but with time, the memory becomes stronger, stable and resilient to disruption, a process known as memory consolidation [18]. The consolidated memory can again become labile if it is reactivated, for example by retrievals and this post-retrieval fragility can return to a stable state through a process known as reconsolidation. Both consolidation and reconsolidation are phases during which memory is vulnerable to interference and during which a strengthening or weakening or disruption of the memory trace can be achieved $[14,18,38,39,63$, 64]. The molecular events underlying consolidation might thus be an extension of the processes that begin during acquisition [38]. Excitatory neurotransmitters are known to be the main neurochemicals involved in the formation of memory, but synapses have differential expressions of neurochemicals [65]. The most abundant excitatory neurotransmitter and the one used at the great majority of fast excitatory synapses in the brain and spinal cord is glutamate and it is said to be very involved in cognitive functions like learning and memory formation $[63,66]$. For learning and memory, glutamate mediates its effects through the activation of two major classes of receptors, the ionotropic (iGluRs) and metabotropic glutamate receptors (mGluRs). Ionotropic glutamate receptors consist of the $\alpha$-amino-3-hydroxy-5methyl-isoxazolepropionic acid receptors (AMPARs), $\mathrm{N}$ methyl-D-aspartate receptors (NMDARs), and kainate receptors [63, 67]. NMDA receptor plays the major role with overwhelming evidence proving its involvement in the actual learning process (encoding), throughout the animal kingdom [66].
Glutamate is also used at most synapses that are capable of increasing or decreasing in strength and are thought to be the main memory storage elements in the brain. Synapses can be persistently potentiated due to strong excitation and remain so for a long period (even for a lifetime) called long-term potentiation (LTP), or remain weak for a long period, due to poor firing or persistent inhibition called long-term depression (LTD). LTP and LTD are enduring changes in synaptic strength, induced by specific patterns of synaptic activity, thought to be cellular models of information storage in the central nervous system, and can also be potentially exploited to treat disorder and disease in the human central nervous system (CNS) [68].

Long-term potentiation (LTP) is key to understanding the cellular and molecular mechanisms by which memories are formed and stored [69]. Hallmark features of LTP are that synaptic changes are associative, rapidly induced, and input specific. Taken together, these characteristics render LTP an attractive model for a cellular basis for learning and memory [63]. Long-term potentiation (LTP) at synapses is classically triggered by the synaptic activation of NMDA receptors (NMDARs) and calcium-permeable (CP) AMPA receptors [70], and it is typically induced by high-frequency tetanic stimulation, which leads to $\mathrm{Na}^{+}$-influx through AMPARs, depolarization of the postsynaptic compartment, and activation of $\mathrm{N}$-methyl-D-aspartate receptors (NMDARs) to permit $\mathrm{Ca}^{2+}$ influx; this sets off a cascade of phosphorylation events to potentiate synaptic transmission [71].

Synaptic plasticity in both excitatory and inhibitory synapses has been found to be dependent upon postsynaptic calcium release which is also dependent on AMPA Rs/ NMDARs system. The dynamic changes in postsynaptic calcium concentration represent the critical elements to determine the direction and amplitude of the changes in synaptic strength, and the activation of several subtypes of glutamate receptors contributes to changes in postsynaptic calcium concentration at synapses, resulting in various types of changes in synaptic strength [67]. Failed regulation of $\mathrm{Ca}^{2+}$ influx through the calcium-permeable (CP) AMPA receptors and NMDARs leads to calcium overload with consequent neuronal hyperexcitability and neurotoxicity which are common phenomena in involuntary motor and sensory disorders.

L-type calcium channels (LTCCs) function to facilitate coupling, mainly excitation-contraction, excitationsecretion, and excitation-transcription, the latter being crucial for neuronal function and memory formation, and under several circumstances, LTCCs have been shown to be important for long-term potentiation (LTP) of synaptic activity [72]. Cav1.2 and Cav1.3 are involved 
in short- and long-term plasticity, but Cav1.3 is the key conductance responsible for short-term sensitization in physiological pain transmission, and Cav1.2 is involved in long-term plasticity associated with neuropathic pain. Therefore, upregulation of Cav1.2 and downregulation of Cav1.3 in neuropathic pain underlies the switch from physiology to pathology [73].

Cav1.2 and Cav1.3 are postsynaptic channels localized predominantly in the soma, spines, and shafts of dendrites that shape neuronal firing, activate $\mathrm{Ca}^{2+}$ signaling pathways involved in excitation-transcription coupling (ETC), and thus regulate neuronal plasticity associated with learning, memory, drug addiction, and neuronal development [30,35]. The importance of LTCCs in protein synthesis-dependent long-term memory is well established and most attention has been given to $\mathrm{CaV}_{1.2}$ for mediating learning [72].

Because of the involvement of $\mathrm{Ca}_{V} 1.2$ and $\mathrm{Ca}_{V} 1.3$ isoforms in synaptic plasticity, synaptic scaling, heterosynaptic molecular dynamics, and transcriptional regulation, it is not surprising that a deficiency of LTCC channels or their increased activity leads to aberrant brain function and neurological disorders [22]. LTCC blockers are known to inhibit both $\mathrm{Ca}_{\mathrm{V}} 1.2$ and $\mathrm{Ca}_{\mathrm{V}} 1.3$ isoforms [74]. Therefore, development of isoformspecific blockers is desirable when there is increased activity of LTCCs. Lamprecht [75] asserted that long-term memory (LTM) formation involves alterations of synaptic efficacy produced by modifications in neural transmission and morphology, and these modifications are mandatory for memory formation. Actin cytoskeleton has been shown to be involved in these key neuronal processes by subserving events such as presynaptic vesicle movement and postsynaptic glutamate and GABA receptor trafficking and dendritic spine morphogenesis, and the actin cytoskeleton and its regulatory proteins are needed for memory formation and extinction in different organisms including mammals. Myosin IIB motor activity regulates NMDAR-driven actin network dynamics and this mechanism is necessary for synaptic plasticity and memory formation [76]. Nonmuscle myosin II (NMII) is a direct regulator of actin polymerization in dendritic spines [77]. It drives synaptic actin polymerization to support plasticity and learning in the hippocampus and basolateral amygdala complex (BLC). Systemic administration of the NMII small molecule inhibitor (NMIIi) blebbistatin (Blebb) produces an immediate, retrieval-independent and long-lasting disruption of the storage of METH-associated memories [17], further confirming the role of nonmuscle myosin II in memory formation.

An array of novel technologies including new molecular-genetic technologies, optical imaging in living animals, and optogenetic tools for manipulation of individual neurons are available to study synapse-specific activity [65, 78-83]. These technologies have provided strong evidence that learning can be associated with the induction of changes in synaptic weights in apparently relevant neural circuits $[40,54]$. It is therefore possible to selectively alter memory in specific cell ensembles at specific areas in the brain.

\section{Formation of maladaptive (reflex) memory}

Normal implicit memory People display certain skills or habits unconsciously and automatically without giving a thought to them. The action happens instantaneously and effortlessly as the demands for them arise. These are usually skills and habits people learned and are accustomed to over time. These can be motor memories of skills and habits involving skeletal muscles such as driving car, playing basketball, playing musical instrument, running, jumping, and so on. After these actions were done repeatedly, and learned overtime, they got saved unconsciously in long-term memories and are retrieved unconsciously and instantaneously when needed.

In the same vein, people unconsciously and automatically differentiate perceptions (somatic, visual, olfactory, gustatory, or auditory) of objects they are accustomed to over time without giving a thought. That is to say, they saved these perceptions unconsciously as different memories in their nervous system in long-term memories. When later in life they encounter these objects, they recall automatically the perceptions of these objects without giving a thought.

The formation of this memory follows normal adaptive process and involves phases of acquisition, consolidation, retrieval, and reconsolidation, and the author believes that the encoding of this type of memory is unconsciously done through interoceptive pathway and consolidated in long-term memory as implicit memory. The encoding is unconscious and the recall is also unconscious and automatic. Even though the recall of this type of memory is automatic and unconscious, it still obeys the "inhibition rule," that is, you can voluntarily stop action if need be.

Abnormal implicit memory A variant of the normal implicit (unconscious) memory can be generated by defective circuit remodeling as a result of physical or chemical injury to the nervous system. When neurons are perturbed by either physical injury or chemical insult, there is usually an attempt to remodel by reestablishing connectivity or regrowth of the neurons, and these cause plastic changes in the neurons. When the process fails or is poorly done, a defect occurs in the circuit. This defect may provoke upregulation of excitatory neurotransmitters (ENTs), mostly involving ionotropic 
glutamate NMDA receptor complexes. The activation of glutamate NMDA receptors would lead to $\mathrm{Ca} 2+$ influx through L-type calcium channels (LTCCs), increased plastic changes in the neurons, and then hyperexcitation of the neurons. Efforts by neurons within affected networks to make homeostatic responses in order to reestablish a proper balance of excitation/inhibition may fail and the homeostatic capacity of the network may be overwhelmed, leading to excitotoxicity. A lot of molecular and structural mechanisms are involved in the processes. The L-type calcium channels (LTCCs) are known to play an important role in triggering intracellular cascades related to synaptic plasticity at glutamatergic synapses [28], and they are involved in normal brain development and plasticity [29]. However, dysregulation of Cav 1.2 and Cav1.3 isoforms of LTCCs (the only LTCCs present in the brain) may lead to calcium overload and have been variously cited to be implicated in pathological circuit remodeling, and manifesting as neuropsychiatric disorders [29-35]. Besides L-type calcium channels (LTCCs) function to facilitate excitationtranscription coupling, modulating neuronal gene transcription and synaptic plasticity [73]. All these are crucial for neuronal function and memory formation. LTCCs have also been shown to be important for longterm potentiation (LTP) of synaptic activity [72]. Because of the involvement of $\mathrm{Ca}_{V} 1.2$ and $\mathrm{Ca}_{V} 1.3$ isoforms in synaptic plasticity, synaptic scaling, heterosynaptic molecular dynamics, and transcriptional regulation, their increased activity leads to aberrant brain function and neurological disorders [22]. NMDA receptor also plays a major role in the actual encoding process.

It is also well known that the plastic changes affect the morphology and number of neurons, followed by increased neuronal activity, expansion of the neuronal receptive field, and hyperexcitability $[9,10,13]$. These events may lead to maladaptive synaptic plasticity, and then formation of maladaptive (reflex) memory which is consolidated with sustained abnormal processes. Because of the increased molecular and structural activities occasioned by these processes, there is augmentation of the memory storage capacity of neurons, as structural rewiring allows more variability and therefore a larger number of potential circuits to be generated, implying a larger memory capacity per synapse [24].

The retrieval phase of the memory is what manifests as involuntary motor and sensory disorders. At this phase, the memory is fragile and unstable. Unlike the normal implicit memory, it has no inhibition control, and therefore remains uncontrollable; hence, it is said to have disobeyed the "inhibition rule." It is the retrieval performance of this memory that defines the uniqueness of these motor and sensory disorders. Again, unlike the normal implicit memory, it does not reconsolidate, and hence remains in persistent recall mode.

Reflex memory theory therefore holds that when this state is sustained for a long time the affected cell ensembles in the network encode this state interoceptively as an aberrant memory that is labile, unstable, and in a "persistent retrieval mode," causing persistence of involuntary and uncontrollable motor and sensory phenomena disorders such as tardive syndromes, flashback and posttraumatic stress disorder, phantom syndromes, and related disorders, in defiance of all available treatments.

Because this memory is in a "persistent retrieval mode, " it is always vulnerable to interferences from pharmacological, molecular, optogenetic, and behavioral manipulations. Figure 3 depicts a highly simplified process of formation of this pathological memory from perturbed neurons, putting side by side with normal implicit memory formation. Figure 4 is a process diagram showing stages of reflex memory formation, and their provoking factors. Potential points of therapeutic interventions have also been proposed as presented in a process diagram (Fig. 5).

Tallet and colleagues [84] capture a picture relating to procedural learning that is applicable to both persistent motor and sensory memories and this has a bearing with reflex memory. That procedural learning follows five successive stages in adults: fast learning, slow learning, consolidation, automatization, and retention. That after repetitive practice followed by further repetitive practice of routines, a consolidation stage occurs where performance undergoes either a spontaneous offline increase in performance or an increase in resistance to interference from the learning of new and competing similar routines. Then, the task is automatized, which means that the new routine can be performed with minimal cognitive resources. Finally, the new routine can be performed with the same level of performance after long delays without further practice (retention stage). Hence, once the new routine has undergone all these five stages, it is robust enough to persist permanently in long-term memory without alteration.

Lüscher and Malenka [85] noted that psychoactive drugs act on glutamatergic and GABAergic synaptic transmission in brain areas causing changes which they refer to as "drug-evoked synaptic plasticity," which outlasts the presence of the drug in the brain and contributes to the reorganization of neural circuits. They further opined that while in most cases early changes are not sufficient to induce disease, with repetitive drug exposure, they may add up and contribute to addictive behavior. The author has adopted this view to say, this is possible only when the effect of the drug is saved in a memory, and in this case, as reflex memory and its recall comes up unconsciously and unpredictably in a reflex 


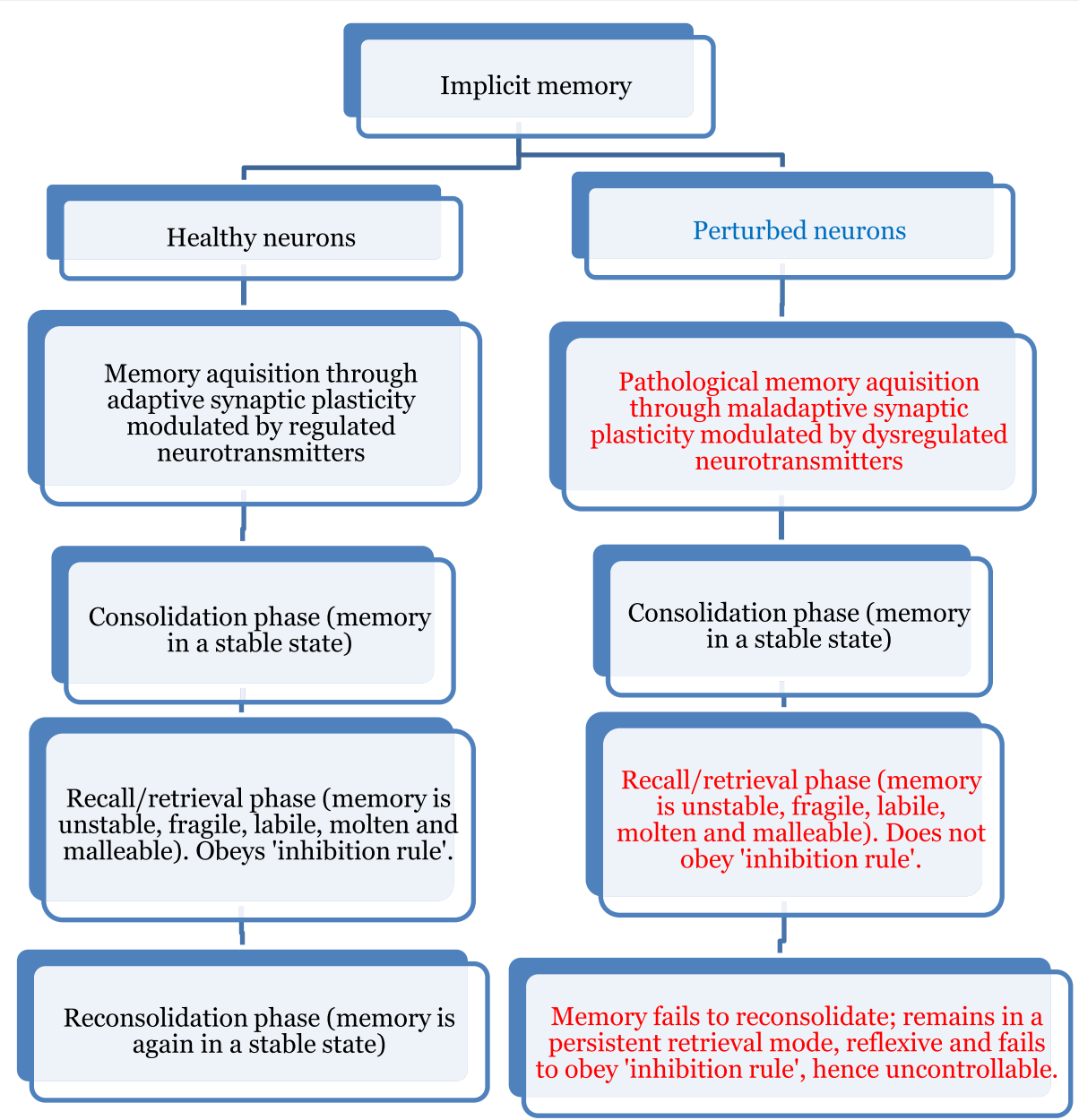

Fig. 3 A process diagram showing how reflex memory is formed as a result of neuronal perturbation. As depicted, healthy memory formation is on the left side, and pathological memory (reflex memory) on the right side. The rectangles marked in red prints indicate potential areas of therapeutic targets. Drugs aimed at inhibiting the formation of, or erasing, the pathological memory may be targeted at phases marked red. Glutamate is most implicated, and upregulated, in neural perturbations of hyperexcitatory nature like TD, PTSDs, phantom syndromes, and similar disorders. Excess glutamate may be mopped up quickly from the synaptic cleft at the memory acquisition phase by enhancing the function of excitatory amino acid transporters (EAATs). Sustained excitotoxicity can lead to calcium overload with consequent neurodegeneration. Cav1.3 isoform-specific blocker may be helpful in any recommended combination therapy. The author thinks that extant theories overlooked the possible role of pathological memory in these phenomena states, and this may have accounted for the resistance of these disorders to extant therapies. A combination therapy that includes memory inhibition and/erasure is therefore advocated

manner and this may explain the "flashback" phenomenon in cocaine addicts or what has been tagged drug memories or drug-associated memories or METHassociated memory or visceral memories [15, 17].

Flashbacks are said to be involuntary, recurrent, intrusive, and vivid images of a trauma that emerge in someone's mind in a waking state, and it is also conceptualized as re-experiencing part of a traumatic event with a realistic intensity as if it were happening in the present [7]. The fact that the "event is reexperienced with a realistic intensity as if it were happening in the present" after a long period of time means a memory of the event was saved. That the recall was "involuntary and intrusive" means that the recall can come up unconsciously and automatically without provocation in a reflex manner. Bourne and colleagues [4] described flashbacks as involuntary memories" which gives credence to the fact that a flashback is a reflex phenomenon.

Similarly, in posttraumatic stress disorder (PTSD), the sensory images of the traumatic scene that happened months or years ago are re-experienced as though they were happening in the present [5].

The same goes for tardive syndromes which often persist for years or even decades after discontinuation of the offending dopamine receptor blocking agents (DRBAs) $[2,3,86,87]$. This can only be possible if the effects of the DRBAs are stored in a memory. 


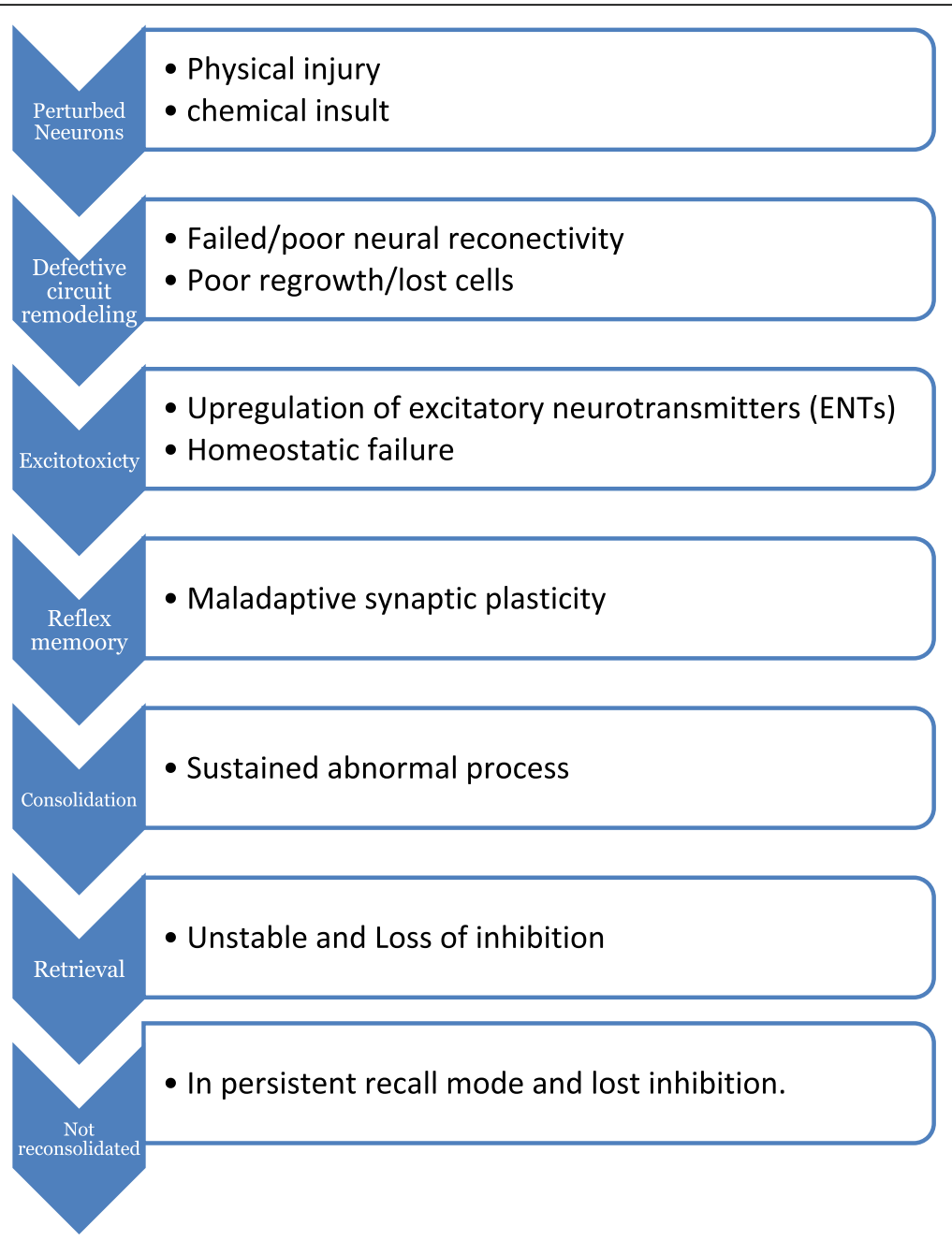

Fig. 4 A process diagram showing stages of reflex memory formation, and their provoking factors. As expressed in the figure, stages of neural events are presented on the left of the diagram and to the right are provoking factors. When neurons are perturbed by either physical injury or chemical insult, there is usually an attempt to remodel by reestablishing connectivity or regrow the neurons. When the process fails or is poorly done, a defect occurs in the circuit. This defect may provoke upregulation of excitatory neurotransmitters (ENTs), mostly ionotropic glutamate NMDA receptor complexes leading to Ca2+ influx and excessive calcium loading, and then excitotoxic stimulation of the neurons with consequent homeostatic failure. Maladaptive synaptic plasticity is induced with consequent formation of abnormal memory, the "reflex memory." Consolidation of the memory occurs as the abnormal process continues. When retrieval or recall occurs, the abnormal memory becomes unstable and fragile. In normal acquired implicit memory, reconsolidation occurs after retrieval, but in this abnormal acquired implicit memory, no reconsolidation occurs because this memory remains in persistent retrieval/recall mode. Because this memory is in a persistent retrieval/recall mode, it is said to have "lost inhibition control" or "disobeyed the inhibition rule." The "loss of inhibition control" is what makes the retrieval uncontrollable. This state of fragility and instability, and persistent recall mode makes this memory vulnerable to interventions using pharmacological, molecular, optogenetic, or behavioral manipulations, and so, can even be erased

The author believes that this pathological form of unconscious memory gives rise to these unconscious, involuntary, and uncontrollable phenomena states when the body synaptic homeostasis fails. Homeostatic plasticity is a negative feedback mechanism that neurons use to offset excessive excitation or inhibition by adjusting their synaptic strengths $[58,88]$, or the ability of a system to return to a set point following perturbation [27]. According to Takeuchi and colleagues [54], it plays both normalizing and protective roles in neural circuits that might otherwise be at risk for seizure through potentiation of too high a proportion of neural afferents. The discussion of homeostatic plasticity in detail is beyond the purpose of this article.

So, the beneficial unconscious memory with its instantaneous auto-recall in a normal state can become disordered as a result of pathological synaptic remodeling coupled with failed synaptic homeostasis to give rise to sensory and motor disorders. The author believes that the defective synaptic remodeling leading to pathological 


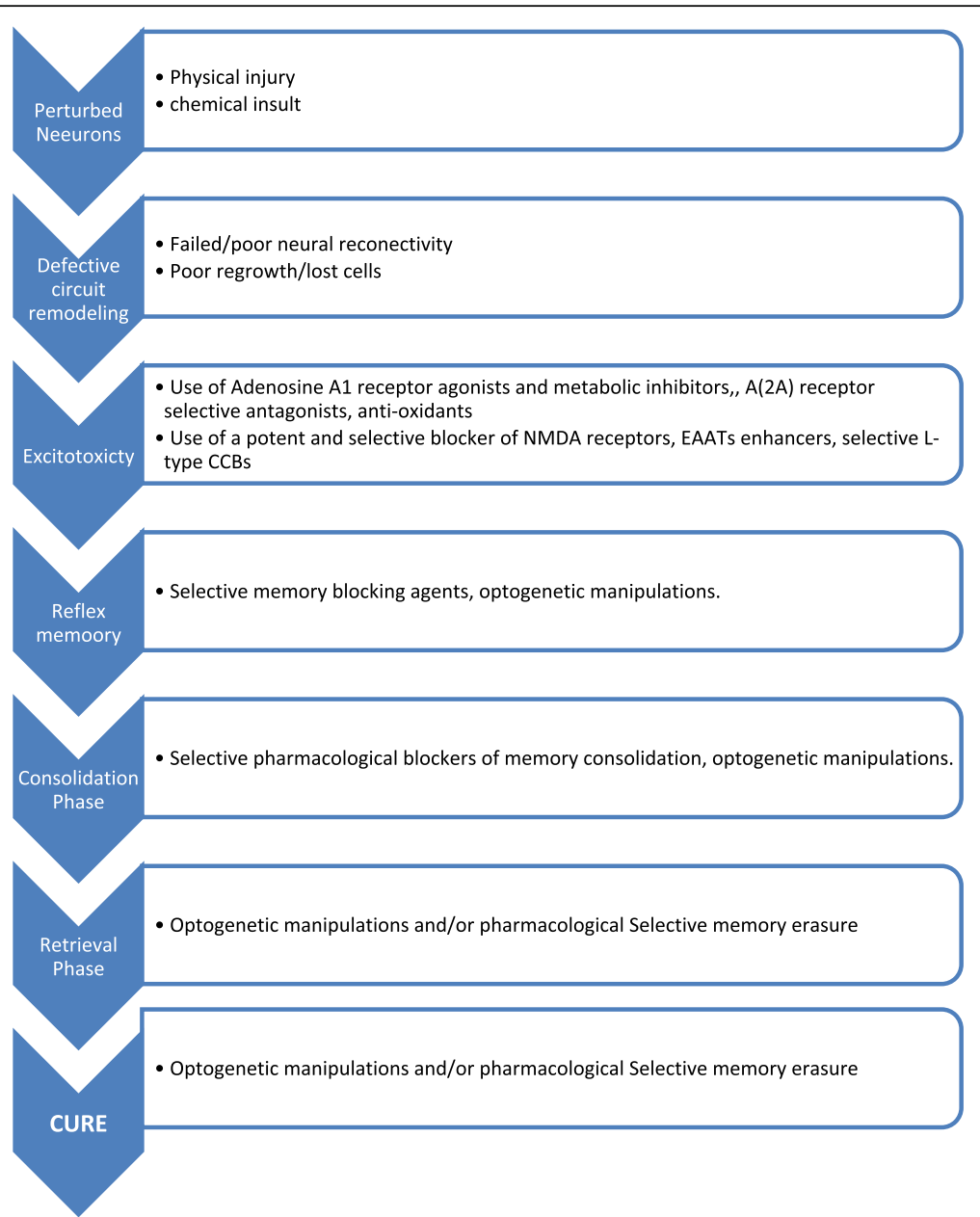

Fig. 5 A process diagram showing stages of the reflex memory formation and points of intervention. As shown, a process diagram has presented the process of reflex memory formation to the left and also proposed interventions to the right. The pathogeneses of acquired excitatory sensory and motor disorders involve multiple molecular and structural mechanisms, and reflex memory only causes persistence of the disorders despite treatment. The proposed points of intervention start from the stage of excitoxicity where there is a need to reduce: excessive neuronal activities; glutamate release; oxidative stress; and calcium loading, and to enhance: glutamate metabolism; and mitochondria integrity. Therefore, use of Adenosine A1 receptor agonists and metabolic inhibitors, A(2A) receptor selective antagonists, antioxidants, potent and selective blockers of NMDA receptors, EAATs enhancers, and selective L-type CCBs are advocated in appropriate rations in the suggested combined treatment protocol of these disorders. At the point of memory formation, selective pharmacological memory blockers (for example, propranolol) and optogenetic manipulations are proposed. At the point that consolidation had occurred, retrieval can be prevented using selective pharmacological blockers and optogenetic manipulations. Retrieval phase follows consolidation phase, and at this point, the memory is fragile and unstable, and quite vulnerable so, the memory can be selectively erased using drugs and optogenetic procedures. Since by its very nature it does not reconsolidate, it remains so vulnerable to various manipulations. If the proposed therapies as explained above are applied, the expected outcome is a cure

memory formation is part of the pathophysiological basis of tardive dyskinesia, phantom phenomena, "flashback," and posttraumatic stress disorder (PTSD) and similar disorders, herein referred to as "acquired reflex phenomena disorders." The pathological memory (reflex memory) is believed to be the end stage of the multiple mechanisms that lead to the generation of these disorders and may be responsible for the persistence of the disorders despite treatment. All extant theories of these disorders failed to recognize this possibility, and it is suspected that this oversight may be the reason why these disorders remained refractory to all extant forms of therapy. This underscores the need to think of therapeutic options that can inhibit formation of, or erase, such abnormal memory.

The author believes too that the memories referred to as drug memories or drug-associated memories or METH-associated memory or visceral memories by researchers [15-17] to explain drug dependence, or proprioceptive memories [13] to explain phantom syndromes, or involuntary memories [4] to explain PTSDs, are all forms of reflex memory caused by pathological synaptic remodeling. What is common to them all is the fact that they are all products of defective circuit remodeling and are all interoceptively encoded. It is 
also assumed that multiple mechanisms involving dysregulation of excitatory neurotransmitters, neurotoxicity, calcium overload, and homeostatic failure give rise to the formation of the memories in $[4,13,15-17]$. It is assumed too that these memories are all labile, fragile, unstable, and in a "persistent recall mode" just like the proposed reflex memory, hence vulnerable to interferences from pharmacological, molecular, optogenetic, and behavioral manipulations. So, treatment options that can work for visceral memories are likely to work for neuroleptic-induced or toxin-induced movement disorders or involuntary sensory disorders due to brain rewiring, because the author believes they all have origin in reflex memory.

\section{Applications of reflex memory theory in the treatment of the disorders}

It is the author's belief that using epigenetic therapies, pharmacotherapuetic approaches, optogenetic manipulations, and other such techniques, people with "acquired reflex phenomena disorders" can be treated with better results than what is obtained presently. This can be achieved using methods involving selective memory erasure or memory inhibition/reversion at specific brain areas, in combination with other extant therapies.

\section{Memory erasure}

Because acquired memory is epigenetically determined, its performance can easily be modulated, whether impaired or improved, by epigenetic processes [89]. Available treatments for neurodegenerative diseases do not arrest disease progression but mainly help in keeping patients from getting worse for a limited period of time. Studies in animal models of neurodegenerative diseases have highlighted the potential role of epigenetic drugs, including inhibitors of histone deacetylases and methyl donor compounds, in ameliorating the cognitive symptoms and preventing or delaying the motor symptoms of the disease, thereby opening the way for a potential application in human pathology [90]. Drugs are available and in use to block or reverse the process through which non-conscious fearful memories of traumatic events become pathological and cause posttraumatic stress disorder (PTSD) and similar debilitating mental illnesses [91]. Several such reports have been recorded where drug memories have been altered and "erased" $[15,17]$.

Professor Barry Everitt and colleagues at the University of Cambridge in 2008 were able to reduce drug-seeking behaviors in rats by blocking a brain chemical receptor important to learning and memory during the recall of drug-associated memories. By disrupting or erasing memories associated with drug use during recall, they could prevent the memories from triggering relapses and drug taking. They opined that when memories are recalled, they become "unstable" or malleable and can be altered or erased during the process of reconsolidation. They further explained that when the animals were given a chemical that interfered with the action of the NMDA-type glutamate receptor (which plays an important role in memory) prior to the "reactivation" session, the rats showed reduced cocaine-seeking behaviors; a single treatment reduced or even stopped drug-seeking behaviors for up to 4 weeks. They expressed hope that this might also be used to treat other neuropsychiatric disorders characterized by maladaptive memories, including posttraumatic stress and phobic anxiety disorders [15]. Bellfy and Kwapis had noted that memory is not a stable record of experience, but instead is an ongoing process that allows existing memories to be modified with new information through a reconsolidation-dependent updating process, and that understanding the molecular mechanisms underlying reconsolidation-dependent memory updating is an important step toward leveraging this process in a therapeutic setting to modify maladaptive memories and to improve memory when it fails [92]

Professor Courtney Miller and colleagues in Florida campus of Scripps Research Institute [17] believed that during neuronal synaptic plasticity memory becomes malleable or exists in a molten state and can be altered or erased. By injecting their experimental rat with a single dose of a modified form of the compound blebbistatin, they selectively erased dangerous addiction-associated memories. They also believed that they were able to achieve this because of the "unstable" state of the "drug memory." It was further explained that normally, actin and nonmuscle myosin II stabilize within minutes of learning, lending stability to long-term memory storage. However, the actin and myosin supporting meth memories behave differently. They remain active and, therefore, uniquely vulnerable to a drug designed to inhibit nonmuscle myosin II [17]. However, Young and colleagues [93] claimed that they produced a long-lasting disruption of context-induced drug seeking (at least 30 days) with a single intra-BLC (basolateral amygdala complex) treatment of blebbistatin (Blebb), an inhibitor of nonmuscle myosin IIB, and that the effect was also independent of retrieval, as METH-associated memory was disrupted $24 \mathrm{~h}$ after a single systemic injection of Blebb delivered in the home cage.

These researchers may have achieved these results because "drug memories" are said to be "unstable" and the author believes these memories are persistently in a "recall mode"/ retrieval mode, and hence vulnerable to interference from the drug blebbistatin. All the researchers that used the drug blebbistatin achieved selective inhibition of nonmuscle myosin IIB (an "upstream" of brain actin) without affecting other long-term memories, such as fear or food reward or motivations and even the formation of new memories. Currently, Blebb is the only small 
molecular inhibitor of the nonmuscle class of myosin II's and is an excellent starting point for medicinal chemistry and a potential substance use disorder (SUD) neurotherapeutic [94]. The drug blebbistatin (Blebb) and optogenetic manipulations used in these studies can be tried for people suffering from acquired reflex phenomena disorders.

\section{Memory inhibition/reversion}

Epigenetic therapy, optogenetic tools, and pharmacological therapies can also be options in this direction. The current epigenetic therapy primarily involves inhibitors of DNA demethylation and histone deacetylation. The great potential for epigenetic therapies lies in the fact that unlike genetic abnormalities, epigenetic changes are reversible, allowing recovery of function for affected genes with normal DNA sequences [95].

Because of the reversible nature of epigenetic events, researchers postulate that inhibition of epigenetic changes could be of valuable therapeutic potential. In fact, in some neurological diseases and cancers, epigenetic drugs are already in use [96]. Mansuy and Mohanna [89] noted that unlike genes, which can be altered only through complex gene therapies, epigenetic marks are reversible and therefore amenable to environmental or drug treatment approaches. They further observed that therapeutic interventions based on drugs appear to be the most direct and rapid treatment strategies, and already in use are inhibitors of enzymes that drive epigenetic processes, such as DNA methyl transferase inhibitors or histone deacetylase (HDAC) inhibitors, in the treatment of cancers like leukemia and lymphoma which could be exploited for treatment of diseases of the central nervous system, such as neurodegenerative and psychiatric diseases, mental retardation, and drug addiction. Therapeutic optogenetic protocols are highly effective at reversing symptoms in animal models of neuropsychiatric disease [82].

Lonergan and colleagues [97] also reported the central inhibitory effects of propranolol on protein synthesis in humans, leading to blockage of memory consolidation and reconsolidation. They further reported that a recent neuroimaging study revealed altered amygdala and hippocampus activity associated with propranolol-induced emotional memory impairment in healthy individuals. Similarly, Terbeck and colleagues [98] reported that propranolol significantly abolished implicit racial bias-automatic negative attitudes in a study involving human subjects. They also noted the key role of the amygdala, and the central effect of propranolol in the process.

\section{Other areas of consideration}

Considering the suspected multiple mechanisms in the pathogeneses of these disorders, a combination therapy is advocated starting from the stage of excitotoxicity. Lglutamate (L-glu) has been consistently implicated in tardive dyskinesia [99, 100], posttraumatic stress disorders [101, 102], and phantom pains/sensations [103105]. Excitotoxicity, a term used in 1969 by Olney to describe the ability of L-glu, as well as structurally related amino acids, to kill nerve cells, a process that has been proposed to take place not only in acute but also chronic diseases of the central nervous system [106]. Excitotoxicity results from the excessive activation of ionotropic glutamate receptors (iGluRs) and leads to a characteristic loss of postsynaptic structures including dendrites and cell bodies [106]. L-glutamate (L-glu) is the major excitatory neurotransmitter in the brain and is functionally involved in virtually all activities of the nervous system; excess extracellular glutamate may lead to excitotoxicity via the overactivation of ionotropic glutamate receptors [106]. Glutamate is metabolized by two major processes: its uptake by glutamate transporters and the subsequent enzymatic degradation by glutamine synthetase [107]. Timely clearance of glutamate from the synaptic cleft is necessary because high levels of extracellular glutamate overactivate glutamate receptors, resulting in excitotoxic effects in the central nervous system [108]. Excitatory amino acid transporters (EAATs) located on the plasma membrane of neurons and glial cells rapidly terminate the action of glutamate and maintain its extracellular concentration below excitotoxic levels [109]. Upon release, glutamate is rapidly taken up by astrocytic glutamate transporters, especially EAAT2 [106], which function to maintain extracellular glutamate levels at low levels [110], but energy depletion and disruption of ionic homeostasis inhibits EAAT activity in astrocytes and may lead to hypo-expression or hypofunction with consequent accumulation of glutamate in the extracellular space [106]. EAAT2 loss or hypofunction contributes to chronic excitotoxicity, and $\beta$ lactam antibiotic ceftriaxone (Cef) has proved to be a positive inducer of EAAT2 in preclinical studies [106].

Sometimes the glutamate excitotoxicity can be independent of ionotropic glutamate receptors, but can be a result of inhibition of the glutamate/cystine antiporter $\mathrm{xc}$, which results in a form of oxidative stress and cell death called oxidative glutamate toxicity which can be inhibited by vitamin E, by group I metabotropic receptor agonists, by a caspase inhibitor, by elevated extracellular cystine, and by the removal of extracellular glutamate [111]. Increased concentrations of extracellular glutamate can also inhibit cystine uptake, leading to glutathione depletion and oxidative glutamate toxicity [108]. There are preclinical and clinical results of the differentiated effectiveness of $\mathrm{N}$-methyl-D-aspartate (NMDA) receptor antagonists, especially antagonists that bind with the GluN2B subunit of NMDA receptors [112, 113].

The L-type calcium channels (LTCCs) are known to play an important role in triggering intracellular 
cascades related to synaptic plasticity at glutamatergic synapses [28], important for long-term potentiation (LTP) of synaptic activity [72], and also function to facilitate excitation-transcription coupling, modulating neuronal gene transcription and synaptic plasticity, with $\mathrm{Ca}_{V} 1.2$ and $\mathrm{Ca}_{V} 1.3$ subunits making distinct contributions to transcriptional responses [73]. Because of the involvement of $\mathrm{Ca}_{\mathrm{V}} 1.2$ and $\mathrm{Ca}_{\mathrm{V}} 1.3$ isoforms in synaptic plasticity, synaptic scaling, heterosynaptic molecular dynamics, and transcriptional regulation, their increased activity leads to aberrant brain function and neurological disorders [22] like the acquired involuntary motor and sensory disorders under investigation. Therefore, use of selective L-type calcium channel blockers (CCBs), especially $\mathrm{Ca}_{\mathrm{V}} 1.2$ and $\mathrm{Ca}_{\mathrm{V}} 1.3$ isoform-specific blockers, may help to attenuate the excitotoxicity.

The increased activities of ionotropic glutamate NMDA receptor complexes with consequent excitotoxocity place a lot of energy demand on synapses. The highenergy demands of synapses lead to high levels of reactive oxygen species (ROS) production, hence oxidative stress. Energy depletion and/or increased oxidative damage to various synaptic proteins can result in a local dysregulation of calcium homeostasis and synaptic degeneration [114]. Mitochondria play a key role in ATP supply to cells via oxidative phosphorylation, and various redox reactions catalyzed by enzymes take place in the oxidative phosphorylation process. An inefficient oxidative phosphorylation may generate reactive oxygen species (ROS), leading to mitochondrial dysfunction [115]. Therefore, there is a need to strengthen the mitochondria integrity by improving the antioxidant defense system, and put up measures to reduce the release of glutamate. Dietary and pharmacological antioxidants have been advocated for, to strengthen antioxidant defense system [114]. Some antioxidants have been studied and they proved effective at preclinical trials and are even undergoing clinical trials now. These include the following: alpha lipoic acid associated with omega-3 [116]; alpha lipoic acid [117]; rice bran oil [118]; Curcumin, the active principle of turmeric (Curcuma longa) [119]; rutin, a polyphenolic flavonoid [120]; caffeine and adenosine [121]; adenosine reuptake/transport inhibitors [122]; Carvedilol [123]; and quercetin, a bioflavonoid [124].

Adenosine receptors modulate neuronal and synaptic function in a range of ways that may make them relevant to the occurrence, development, and treatment of brain ischemic damage and degenerative disorders. $\mathrm{A}(1)$ adenosine receptors tend to suppress neural activity by a predominantly presynaptic action, while $\mathrm{A}(2 \mathrm{~A})$ adenosine receptors are more likely to promote transmitter release and postsynaptic depolarization [125]. Both agonists of adenosine $\mathrm{A}(1)$ and $\mathrm{A}(2)$ receptors and the antagonists of $\mathrm{A}(2 \mathrm{~A})$ receptors are known to protect against neuronal damage caused by toxins as well as they can also protect against the cell damage inflicted by reactive oxygen species [121], and so, can be used as antioxidants. Adenosine reuptake inhibitors dipyridamole and nimodipine (L-type channel calcium antagonist), an adenosine transport inhibitor, could be possible therapeutic antioxidants [122]. Adenosine is an endogenous neuroprotective metabolite, and in situations of metabolic stress, adenosine decreases energy demand and increases energy supply; in this context, it modulates glutamate release. This shift of adenosine-glutamate balance in favor of adenosine helps to restore function at the cellular, organ, and organism level [126]. Adenosine agonists can therefore help in reducing excessive glutamate release at the stage of excitotoxicity. Adenosine increases dramatically during enhanced nerve activity, hypoxia, or ischemia, and in these pathological conditions, adenosinergic transmission-potentiating agents, which elevate adenosine level by either inhibiting its degradation (adenosine deaminase and kinase inhibitors) or preventing its transport, offer protection against ischemic or excitotoxic neuronal damage; the directly acting adenosine A1 receptor agonists are known to mediate neuroprotection, mostly by the blockade of $\mathrm{Ca} 2+$ influx, which results in the inhibition of glutamate release and reduction of its excitatory effects at a postsynaptic level [127]. Antagonists of adenosine A2A receptors markedly reduce cerebral ischemic damage in animal models of focal and global ischemia by attenuating the neuronal loss induced by excitatory amino acids (EAA). Hence, it might be suggested that adenosine A2A receptor antagonists may represent a novel strategy in the therapeutic approach to pathologies characterized by acute or chronic neurodegenerative events [127]. Presynaptic release of the excitotoxic neurotransmitters glutamate and aspartate impair intracellular $\mathrm{Ca} 2+$ homeostasis or induce uncontrolled membrane depolarization leading to cytotoxic calcium loading and neuronal cell death [128], but adenosine counteracts this process by inhibiting neuronal Ca2+ influx [128, 129]. In addition, adenosine directly stabilizes the neuronal membrane potential by increasing the conductance for $\mathrm{K}+$ and $\mathrm{Cl}-$ ions, thereby counteracting excessive membrane depolarization [128]. It is therefore justifiable using a combined therapy of antioxidants, adenosine agonists and metabolic inhibitors, selective blockers of NMDA receptors, EAAT2 enhancers, and selective Ltype calcium channel blockers (CCBs) to reduce the excitotoxic effects of the excitatory amino acids (EAAs). Recently, two prospective epidemiological studies of large cohorts have firmly established the beneficial effects of consumption of caffeine (a non-specific adenosine antagonist) as a neuroprotector for mediating 
neuroprotection in a variety of brain injuries induced by stroke, excitotoxicity, and mitochondrial toxins [130]. Therefore, it may be worth putting on clinical trials these agents, using people with involuntary motor and sensory disorders.

\section{Conclusions}

Acquired involuntary motor and sensory disorders originate from defective circuit remodeling but involve multiple neural mechanisms. Dysregulation of excitatory neurotransmitters, neurotoxicity, calcium overload, and homeostatic failure is implicated in the process. Sustained effects of these defective mechanisms are encoded interoceptively as a memory (the reflex memory) in the neurons. The involuntary motor and sensory phenomena are the conscious manifestations of this memory. Unlike the normal implicit memory, it has no inhibition control, and therefore remains uncontrollable; hence, it is said to have disobeyed the "inhibition rule." It is the retrieval performance of this memory that defines the uniqueness of these motor and sensory disorders. This memory is proposed to be responsible for the persistence of tardive dyskinesia, flashbacks, and posttraumatic stress disorders, phantom pains and sensations, and related disorders even with all known therapies. All extant theories of these disorders failed to recognize this possibility, and it is suspected that this oversight may be the reason why these disorders remained refractory to all extant forms of therapy. A combination therapy that includes memory erasure and memory inhibition/reversion is expected to give a cure.

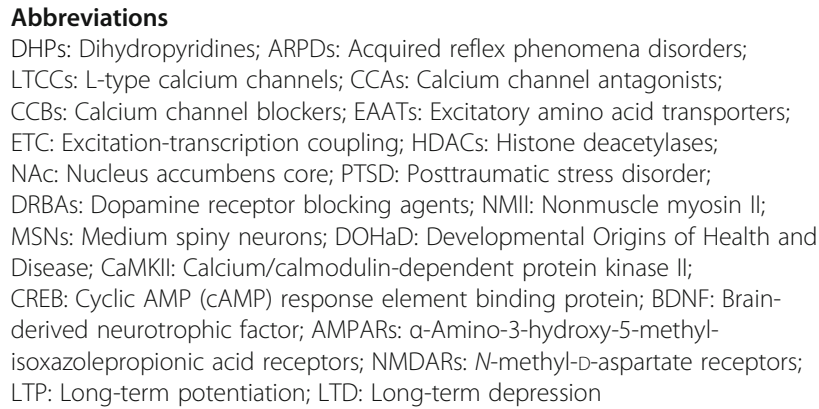

\section{Acknowledgements}

I want to thank Professor Peter Agogo of faculty of Education Benue State University for proofreading the manuscript, and for his permission to be acknowledged in this publication.

\section{Author's contributions}

The author solely developed the concept and wrote the manuscript including the typesetting. The author read and approved the final manuscript.

\section{Author's information}

The author is a practicing Psychiatrist at Federal Medical Centre, Makurdi, Benue State, Nigeria.

\section{Funding}

This work is self-funded.
Availability of data and materials

Not applicable

\section{Declarations}

Ethics approval and consent to participate

Not applicable

Consent for publication

Not applicable

\section{Competing interests}

The author declares that there are no competing interests.

Received: 15 September 2020 Accepted: 14 April 2021

Published online: 17 May 2021

\section{References}

1. Egan MF, Apud J, Wyatt RJ. Treatment of tardive dyskinesia. Schizophr Bull. 1997;23:583-609.

2. Kulkarni SK, Naidu PS. Pathophysiology and drug therapy of tardive dyskinesia: current concepts and future perspectives. Drugs Today (Barc). 2003:39:19-49.

3. Peckham AM, Nicewonder JA. VMAT2 inhibitors for tardive dyskinesiapractice implications. J Pharm Pract. 2019:32(4):450-7.

4. Bourne C, Mackay CE, Holmes EA. The neural basis of flashback formation: the impact of viewing trauma. Psychol Med. 2013;43(7):1521-32.

5. Whalley MA, Kroes MCW, Huntley Z, Rugg MD, Davis SW, Brewin CR. An fMRI investigation of posttraumatic flashbacks. Brain Cogn. 2013;81:151-9.

6. Bailey CR, Cordell E, Sobin SM, Neumeister A. Recent progress in understanding the pathophysiology of post-traumatic stress disorder: implications for targeted pharmacological treatment. CNS Drugs. 2013;27(3): 221-32.

7. Kummer A, Harsanyi E. Flashbacks in social anxiety disorder: psychopathology of a case. Indian J Psychiatry. 2008;50:200-1.

8. Ribbers G, Mulder T, Rijken R. The phantom phenomenon: a critical review. Int J Rehabil Res. 1989;12:175-86.

9. Flor H. Phantom-limb pain: characteristics, causes, and treatment. Lancet Neurol. 2002;1(3):182-9.

10. Subedi B, Grossberg GT. Phantom limb pain: mechanisms and treatment approaches. Pain Res Treat. 2011;2011:864605.

11. Björkman B, Lund I, Arnér S, Hydén T. Phantom phenomena - their perceived qualities and consequences from the patient's perspective. Scand J Pain. 2012;3:134-40.

12. Pirowska A, Wloch T, Nowobilski R, Plaszewski M, Hocini A, Ménager D. Phantom phenomena and body scheme after limb amputation: a literature review. Neurol Neurochir Pol. 2014:48(1):52-9.

13. Collins KL, Russell HG, Schumacher PJ, Robinson-Freeman KE, O'Conor EC, Gibney KD, et al. A review of current theories and treatments for phantom limb pain. J Clin Invest. 2018;128:2168-76.

14. Merlo E, Bekinschtein P, Jonkman S, Medina JH. Molecular mechanisms of memory consolidation, reconsolidation, and persistence. Neural Plast. 2015; 2015:687175.

15. Milton AL, Lee JLC, Butler VJ, Gardner R, Everitt BJ. Intra-amygdala and systemic antagonism of NMDA receptors prevents the reconsolidation of drug-associated memory and impairs subsequently both novel and previously acquired drug-seeking behaviors. JNEUROSCI. 2008;28:8230-7.

16. Kuijer EJ, Ferragud A, Milton AL. Retrieval-extinction and relapse prevention: rewriting maladaptive drug memories? Front Behav Neurosci. 2020;14:23.

17. Briggs SB, Hafenbreidel M, Young EJ, Rumbaugh G, Miller CA. The role of nonmuscle myosin II in polydrug memories and memory reconsolidation. Learn Mem. 2018;25(9):391-8.

18. Bisaz R, Travaglia A, Alberini CM. The neurobiological bases of memory formation: from physiological conditions to psychopathology. Psychopathology. 2014:47(6):347-56.

19. Ehlers A. Understanding and treating unwanted trauma memories in posttraumatic stress disorder. Z Psychol. 2010;218(2):141-5.

20. Yaron A, Schuldiner O. Common and divergent mechanisms in developmental neuronal remodeling and dying back neurodegeneration. Curr Biol. 2016;26:R628-39. 
21. Bradley PM, Denecke CK, Aljovic A, Schmalz A, Kerschensteiner M, Bareyre FM. Corticospinal circuit remodeling after central nervous system injury is dependent on neuronal activity. J Exp Med. 2019;216:2503-14.

22. Stanika Rl, Flucher BE, Obermair GJ. Regulation of postsynaptic stability by the L-type calcium channel CaV1.3 and its interaction with PDZ proteins. Curr Mol Pharmacol. 2015;8(1):95-101.

23. Roig-Puiggros S, Vigouroux RJ, Beckman D, Bocai NI, Chiou B, Davimes J, et al. Construction and reconstruction of brain circuits: normal and pathological axon guidance. J Neurochem. 2020;153:10-32.

24. Kim W, Kim SK. Neural circuit remodeling and structural plasticity in the cortex during chronic pain. Korean J Physiol Pharmacol. 2016;20:1-8.

25. Kim SK, Nabekura J. Rapid synaptic remodeling in the adult somatosensory cortex following peripheral nerve injury and its association with neuropathic pain. J Neurosci. 2011;31(14):5477-82.

26. Eto K, Ishikawa T, Sun Kwang K, Nabekura J. Mechanisms of neural circuit remodeling in the primary somatosensory cortex in mouse models of chronic pain. Brain Nerve. 2013;65(6):623-33.

27. Ramocki MB, Zoghbi HY. Failure of neuronal homeostasis results in common neuropsychiatric phenotypes. Nature. 2008;455:912-8.

28. Yuan Q, Shakhawat AM, Harley CW. Mechanisms underlying early odor preference learning in rats. Prog Brain Res. 2014;208:115-56.

29. Kabir ZD, Martínez-Rivera A, Rajadhyaksha AM. From gene to behavior: Ltype calcium channel mechanisms underlying neuropsychiatric symptoms. Neurotherapeutics. 2017;14:588-613.

30. Striessnig J, Ortner NJ, Pinggera A. Pharmacology of L-type calcium channels: novel drugs for old targets? Curr Mol Pharmacol. 2015;8:110-22.

31. Bhat S, Dao DT, Terrillion CE, Arad M, Smith RJ, Soldatov NM, et al. CACN A1C (Cav1.2) in the pathophysiology of psychiatric disease. Prog Neurobiol. 2012;99:1-14.

32. Casamassima F, Hay AC, Benedetti A, Lattanzi L, Cassano GB, Perlis RH. Ltype calcium channels and psychiatric disorders: a brief review. Am J Med Genet B Neuropsychiatr Genet. 2010;153B:1373-90.

33. Moon AL, Haan N, Wilkinson LS, Thomas KL, Hall J. CACNA1C: Association with psychiatric disorders, behavior, and neurogenesis. Schizophr Bull. 2018; 44:958-65.

34. Martínez-Rivera A, Hao J, Tropea TF, Giordano TP, Kosovsky M, Rice RC, et al. Enhancing VTA Ca 1.3 L-type $\mathrm{Ca}^{2+}$ channel activity promotes cocaine and mood-related behaviors via overlapping AMPA receptor mechanisms in the nucleus accumbens. Mol Psychiatry. 2017;22:1735-45.

35. Pinggera A, Lieb A, Benedetti B, Lampert M, Monteleone S, Liedl KR, et al. CACNA1D de novo mutations in autism spectrum disorders activate Cav1.3 L-type calcium channels. Biol. Psychiatry. 2015;77:816-22.

36. Lüthi $A$, Lüscher C. Pathological circuit function underlying addiction and anxiety disorders. Nat Neurosci. 2014;17:1635-43.

37. Kornhuber J, Zoicas I. Social fear memory requires two stages of protein synthesis in mice. Int J Mol Sci. 2020;21(15):5537.

38. Abel T, Lattal KM. Molecular mechanisms of memory acquisition, consolidation and retrieval. Curr Opin Neurobiol. 2001;11:180-7.

39. Szapiro G, Galante JM, Barros DM, Levi de Stein M, Vianna MR, Izquierdo LA, et al. Molecular mechanisms of memory retrieval. Neurochem Res. 2002;27: 1491-8.

40. Hoshiba Y, Wada T, Hayashi-Takagi A. Synaptic ensemble underlying the selection and consolidation of neuronal circuits during learning. Front Neural Circuits. 2017;11:12

41. Thompson RF, Kim JJ. Memory systems in the brain and localization of a memory. PNAS. 1996;93(24):13438-44.

42. Squire LR, Dede AJ. Conscious and unconscious memory systems. Cold Spring Harb Perspect Biol. 2015;7(3):a021667.

43. Manns JR, Squire LR. Perceptual learning, awareness, and the hippocampus. Hippocampus. 2001;11(6):776-82.

44. Proske U, Gandevia SC. The proprioceptive senses: their roles in signaling body shape, body position and movement, and muscle force. Physiol Rev. 2012;92(4):1651-97.

45. Hirst W, Phelps EA. Flashbulb Memories. Curr Dir Psychol Sci. 2016;25:36-41.

46. Bateson P, Barker D, Clutton-Brock T, Deb D, D'Udine B, Foley RA, et al. Developmental plasticity and human health. Nature. 2004;430:419-21.

47. Gluckman PD, Hanson MA. Developmental plasticity and human disease: research directions. J Int Med. 2007;261:461-71.

48. Moczek AP, Sultan S, Foster S, Ledón-Rettig C, Dworkin I, Nijhout HF, et al. The role of developmental plasticity in evolutionary innovation. Proc Biol Sci. 2011;278:2705-13.
49. Moosavi A, Ardekani AM. Role of epigenetics in biology and human diseases. Iran Biomed J. 2016:20:246-58.

50. Heindel JJ, Vandenberg LN. Developmental origins of health and disease: a paradigm for understanding disease cause and prevention. Curr Opin Pediatr. 2015;27:248-53.

51. Heindel JJ, Balbus J, Birnbaum L, Brune-Drisse MN, Grandjean P, Gray K, et al. Developmental origins of health and disease: integrating environmental influences. Endocrinology. 2015;156(10):3416-21.

52. Tang WY, Ho SM. Epigenetic reprogramming and imprinting in origins of disease. Rev Endocr Metab Disord. 2007;8:173-82.

53. Nirenberg M. Genetic Memory. JAMA. 1968;206(9):1973-7.

54. Takeuchi T, Duszkiewicz AJ, Morris RG. The synaptic plasticity and memory hypothesis: encoding, storage and persistence. Philos Trans R Soc Lond Ser B Biol Sci. 2013;369(1633):20130288.

55. Ceunen E, Vlaeyen JWS, Diest IV. On the origin of interoception. Front Psychol. 2016;7:743.

56. Price CJ, Hooven C. Interoceptive awareness skills for emotion regulation: theory and approach of mindful awareness in body-oriented therapy (MABT). Front Psychol. 2018;9:798.

57. Cameron O. Interoception: the inside story - a model for psychosomatic processes. Psychosom Med. 2001;63(5):697-710.

58. Pozo K, Goda Y. Unraveling mechanisms of homeostatic synaptic plasticity. Neuron. 2010:66:337-51.

59. Herculano-Houzel S. The remarkable, yet not extraordinary, human brain as a scaled-up primate brain and its associated cost. PNAS. 2012; 109(Supplement 1):10661-8.

60. Tong MT, Peace ST, Cleland TA. Properties and mechanisms of olfactory learning and memory. Front Behav Neurosci. 2014;8:238.

61. Viho EMG, Buurstede JC, Mahfouz A, Koorneef LL, van Weert L. TCM, Houtman R, et al. Corticosteroid action in the brain: the potential of selective receptor modulation. Neuroendocrinology. 2019;109:266-76.

62. Barsegyan A, Mackenzie SM, Kurose BD, McGaugh JL, Roozendaal B. Glucocorticoids in the prefrontal cortex enhance memory consolidation and impair working memory by a common neural mechanism. Proc Natl Acad Sci U S A. 2010;107:16655-60.

63. Gal-Ben-Ari S, Rosenblum K. Molecular mechanisms underlying memory consolidation of taste information in the cortex. Front Behav Neurosci. 2012;5:87.

64. Bailey $\mathrm{CH}$, Kandel ER, Harris KM. Structural components of synaptic plasticity and memory consolidation. Cold Spring Harb Perspect Biol. 2015;7(7): a021758.

65. Kohl MM, Shipton OA, Deacon RM, Rawlins JN, Deisseroth K, Paulsen O. Hemisphere-specific optogenetic stimulation reveals left-right asymmetry of hippocampal plasticity. Nat Neurosci. 2011;14:1413-5

66. Riedel G, Platt B, Micheau J. Glutamate receptor function in learning and memory. Behav Brain Res. 2003;140:1-47.

67. Greget R, Pernot F, Bouteiller JM, Ghaderi V, Allam S, Keller AF, et al. Simulation of postsynaptic glutamate receptors reveals critical features of glutamatergic transmission. PLoS One. 2011;6(12):e28380.

68. Bliss TVP, Cooke SF. Long-term potentiation and long-term depression: a clinical perspective. Clinics (Sao Paulo). 2011;66(Suppl 1):3-17.

69. Leite JP, Neder L, Arisi GM, Carlotti CG Jr, Assirati JA, Moreira JE. Plasticity, synaptic strength, and epilepsy: what can we learn from ultrastructural data? Epilepsia. 2005;46(Suppl 5):134-41.

70. Park P, Kang H, Sanderson TM, Bortolotto ZA, Georgiou J, Zhuo M, et al. The role of calcium-permeable AMPARs in long-term potentiation at principal neurons in the rodent hippocampus. Front Synaptic Neurosci. 2018;10:42.

71. Chater TE, Goda Y. The role of AMPA receptors in postsynaptic mechanisms of synaptic plasticity. Front Cell Neurosci. 2014;8:401.

72. Ghosh A, Carew SJ, Chen X, Yuan Q. The role of L-type calcium channels in olfactory learning and its modulation by norepinephrine. Front Cell Neurosci. 2017;11:394.

73. Radwani H, Lopez-Gonzalez MJ, Cattaert D, Roca-Lapirot O, Dobremez E, Bouali-Benazzouz R, et al. Cav1.2 and Cav1.3 L-type calcium channels independently control short- and long-term sensitization to pain. J Physiol. 2016:594(22):6607-26

74. Striessnig J, Koschak A, Sinnegger-Brauns MJ, Hetzenauer A, Nguyen NK, Busquet $P$, et al. Role of voltage-gated L-type Ca2+ channel isoforms for brain function. Biochem Soc Trans. 2006;34(Pt 5):903-9.

75. Lamprecht R. The actin cytoskeleton in memory formation. Prog Neurobiol. 2014;117:1-19. 
76. Rex CS, Gavin CF, Rubio MD, Kramar EO, Chen LY, Jia Y, et al. Myosin IIB regulates actin dynamics during synaptic plasticity and memory formation. Neuron. 2010;67:603-17.

77. Briggs SB, Blouin AM, Young EJ, Rumbaugh G, Miller CA. Memory disrupting effects of nonmuscle myosin II inhibition depend on the class of abused drug and brain region. Learn Mem. 2017;24:70-5.

78. Carter ME, de Lecea L. Optogenetic investigation of neural circuits in vivo. Trends Mol Med. 2011;17:197-206.

79. Goold CP, Nicoll RA. Single-cell optogenetic excitation drives homeostatic synaptic depression. Neuron. 2010;68:512-28.

80. Han X. In vivo application of optogenetics for neural circuit analysis. ACS Chem Neurosci. 2012;3:577-84

81. Xie YF, Jackson MF, Macdonald JF. Optogenetics and synaptic plasticity. Acta Pharmacol Sin. 2013;34:1381-5.

82. Creed M, Pascoli VJ, Lüscher C. Refining deep brain stimulation to emulate optogenetic treatment of synaptic pathology. Science. 2015;347:659-64.

83. Ordaz JD, Wu W, Xu XM. Optogenetics and its application in neural degeneration and regeneration. Neural Regen Res. 2017;12:1197-209.

84. Tallet J, Albaret JM, Rivière J. The role of motor memory in action selection and procedural learning: insights from children with typical and atypical development. Socioaffect Neurosci Psychol. 2015;5:28004.

85. Lüscher C, Malenka RC. Drug-evoked synaptic plasticity in addiction: from molecular changes to circuit remodeling. Neuron. 2011;69:650-63.

86. Lerner PP, Miodownik C, Lerner V. Tardive dyskinesia (syndrome): current concept and modern approaches to its management. Psychiatry Clin Neurosci. 2015;69(6):321-34.

87. Teo JT, Edwards MJ, Bhatia K. Tardive dyskinesia is caused by maladaptive synaptic plasticity: a hypothesis. Mov Disord. 2012;27(10):1205-15.

88. Turrigiano G. Homeostatic synaptic plasticity: local and global mechanisms for stabilizing neuronal function. Cold Spring Harb Perspect Biol. 2012;4(1): a005736.

89. Mansuy IM, Mohanna S. Epigenetics and the human brain: where nurture meets nature. Cerebrum. 2011;2011:8.

90. Coppedè $F$. The potential of epigenetic therapies in neurodegenerative diseases. Front Genet. 2014;5:220.

91. Glannon W. Psychopharmacology and memory. J Med Ethics. 2006;32(2):74-8.

92. Bellfy L, Kwapis JL. Molecular mechanisms of reconsolidation-dependent memory updating. Int J Mol Sci. 2020;21(18):6580.

93. Young EJ, Blouin AM, Briggs SB, Sillivan SE, Lin L, Cameron MD, et al. Nonmuscle myosin IIB as a therapeutic target for the prevention of relapse to methamphetamine use. Mol Psychiatry. 2016;21(5):615-23.

94. Young EJ, Briggs SB, Rumbaugh G, Miller CA. Nonmuscle myosin II inhibition disrupts methamphetamine-associated memory in females and adolescents. Neurobiol Learn Mem. 2017;139:109-16.

95. Ahuja N, Sharma AR, Baylin SB. Epigenetic therapeutics: a new weapon in the war against cancer. Annu Rev Med. 2016;67:73-89.

96. Heerboth S, Lapinska K, Snyder N, Leary M, Rollinson S, Sarkar S. Use of epigenetic drugs in disease: an overview. Genet Epigenet. 2014;6:9-19.

97. Lonergan MH, Olivera-Figueroa LA, Pitman RK, Brunet A. Propranolol's effects on the consolidation and reconsolidation of long-term emotional memory in healthy participants: a meta-analysis. J Psychiatry Neurosci. 2013; 38:222-31.

98. Terbeck S, Kahane G, McTavish S, Savulescu J, Cowen PJ, Hewstone M. Propranolol reduces implicit negative racial bias. Psychopharmacology. 2012:222:419-24.

99. Gerlach J. Tardive dyskinesia. Pathophysiological mechanisms and clinical trials. Encephale. 1988;14 Spec No:227-32.

100. Tsai G, Goff DC, Chang RW, Flood J, Baer L, Coyle JT. Markers of glutamatergic neurotransmission and oxidative stress associated with tardive dyskinesia. Am J Psychiatry. 1998;155(9):1207-13.

101. Chambers RA, Bremner JD, Moghaddam B, Southwick SM, Charney DS, Krystal JH. Glutamate and post-traumatic stress disorder: toward a psychobiology of dissociation. Semin Clin Neuropsychiatry. 1999;4(4): 274-81.

102. Averill LA, Purohit P, Averill CL, Boesl MA, Krystal JH, Abdallah CG. Glutamate dysregulation and glutamatergic therapeutics for PTSD: Evidence from human studies. Neurosci Lett. 2017;649:147-55.

103. Wei F, Li P, Zhuo M. Loss of synaptic depression in mammalian anterior cingulate cortex after amputation. J Neurosci. 1999;19(21):9346-54.

104. Zhuo M. Glutamate receptors and persistent pain: targeting forebrain NR2B subunits. Drug Discov Today. 2002;7(4):259-67.
105. Wu MF, Pang ZP, Zhuo M, Xu ZC. Prolonged membrane potential depolarization in cingulate pyramidal cells after digit amputation in adult rats. Mol Pain. 2005;1:23

106. Lewerenz J, Maher P. Chronic glutamate toxicity in neurodegenerative diseases-what is the evidence? Front Neurosci. 2015;9:469.

107. Ishikawa M. Abnormalities in glutamate metabolism and excitotoxicity in the retinal diseases. Scientifica (Cairo). 2013;2013:528940.

108. Wang J, Wang F, Mai D, Qu S. Molecular mechanisms of glutamate toxicity in Parkinson's disease. Front Neurosci. 2020;14:585584.

109. Shigeri Y, Seal RP, Shimamoto K. Molecular pharmacology of glutamate transporters, EAATs and VGLUTs. Brain Res Brain Res Rev. 2004;45(3):250-65.

110. Todd AC, Hardingham GE. The regulation of astrocytic glutamate transporters in health and neurodegenerative diseases. Int J Mol Sci. 2020; 21(24):9607.

111. Schubert D, Piasecki D. Oxidative glutamate toxicity can be a component of the excitotoxicity cascade. J Neurosci. 2001;21(19):7455-62.

112. Szota AM, Scheel-Krüger J. The role of glutamate receptors and their interactions with dopamine and other neurotransmitters in the development of tardive dyskinesia: preclinical and clinical results. Behav Pharmacol. 2020;31(6):511-23.

113. Naidu PS, Kulkarni SK. Excitatory mechanisms in neuroleptic-induced vacuous chewing movements (VCMs): possible involvement of calcium and nitric oxide. Behav Pharmacol. 2001;12(3):209-16.

114. Mattson MP, Liu D. Energetics and oxidative stress in synaptic plasticity and neurodegenerative disorders. NeuroMolecular Med. 2002; 2(2):215-31.

115. Singh A, Kukreti R, Saso L, Kukreti S. Oxidative Stress: A Key Modulator in Neurodegenerative Diseases. Molecules. 2019;24(8):1583.

116. de Araújo DP, Camboim TGM, Silva APM, Silva CDF, de Sousa RC, Barbosa MDA, et al. Behavioral and neurochemical effects of alpha lipoic acid associated with omega-3 in tardive dyskinesia induced by chronic haloperidol in rats. Can J Physiol Pharmacol. 2017;95(7):837-43.

117. Thaakur S, Himabindhu G. Effect of alpha lipoic acid on the tardive dyskinesia and oxidative stress induced by haloperidol in rats. J Neural Transm (Vienna). 2009;116(7):807-14.

118. Samad N, Haleem DJ. Antioxidant effects of rice bran oil mitigate repeated haloperidol-induced tardive dyskinesia in male rats. Metab Brain Dis. 2017; 32(4):1099-107.

119. Bishnoi M, Chopra K, Kulkarni SK. Protective effect of Curcumin, the active principle of turmeric (Curcuma longa) in haloperidol-induced orofacial dyskinesia and associated behavioural, biochemical and neurochemical changes in rat brain. Pharmacol Biochem Behav. 2008; 88(4):511-22

120. Bishnoi M, Chopra K, Kulkarni SK. Protective effect of rutin, a polyphenolic flavonoid against haloperidol-induced orofacial dyskinesia and associated behavioural, biochemical and neurochemical changes. Fundam Clin Pharmacol. 2007;21(5):521-9.

121. Bishnoi M, Chopra K, Kulkarni SK. Involvement of adenosinergic receptor system in an animal model of tardive dyskinesia and associated behavioural, biochemical and neurochemical changes. Eur J Pharmacol. 2006;552(1-3): 55-66.

122. Bishnoi M, Chopra K, Kulkarni SK. Protective effect of adenosine reuptake inhibitors in haloperidol-induced orofacial dyskinesia and associated behavioural, biochemical and neurochemical changes. Pharmacology. 2007; 79(3):171-83.

123. Naidu PS, Singh A, Kulkarni SK. Carvedilol attenuates neuroleptic-induced orofacial dyskinesia: possible antioxidant mechanisms. Br J Pharmacol. 2002; 136(2):193-200

124. Naidu PS, Singh A, Kulkarni SK. Quercetin, a bioflavonoid, attenuates haloperidol-induced orofacial dyskinesia. Neuropharmacology. 2003;44(8): 1100-6.

125. Stone TW, Ceruti S, Abbracchio MP. Adenosine receptors and neurological disease: neuroprotection and neurodegeneration. Handb Exp Pharmacol. 2009;193:535-87.

126. Deckert J, Gleiter CH. Adenosine--an endogenous neuroprotective metabolite and neuromodulator. J Neural Transm Suppl. 1994;43:23-31.

127. Wardas J. Neuroprotective role of adenosine in the CNS. Pol J Pharmacol. 2002;54(4):313-26

128. Rudolphi KA, Schubert P, Parkinson FE, Fredholm BB. Adenosine and brain ischemia. Cerebrovasc Brain Metab Rev. 1992;4(4):346-69. 
129. Abbracchio MP, Cattabeni F. Brain adenosine receptors as targets for therapeutic intervention in neurodegenerative diseases. Ann N Y Acad Sci. 1999;890:79-92.

130. Kalda A, Yu L, Oztas E, Chen JF. Novel neuroprotection by caffeine and adenosine $A(2 A)$ receptor antagonists in animal models of Parkinson's disease. J Neurol Sci. 2006;248(1-2):9-15.

\section{Publisher's Note}

Springer Nature remains neutral with regard to jurisdictional claims in published maps and institutional affiliations.

Submit your manuscript to a SpringerOpen ${ }^{\circ}$ journal and benefit from:

- Convenient online submission

- Rigorous peer review

- Open access: articles freely available online

High visibility within the field

- Retaining the copyright to your article

Submit your next manuscript at $\boldsymbol{\nabla}$ springeropen.com 